\title{
Discrimination of Green, Oolong, and Black Teas by GC-MS Analysis of Characteristic Volatile Flavor Compounds
}

\author{
Susanne Baldermann"1,2, Ziyin Yang3, Tsuyoshi Katsuno4, Vo Anh Tu5, Nobuyuki Mase6, \\ Yoriyuki Nakamura ${ }^{4}$, Naoharu Watanabe ${ }^{5,7 *}$ \\ ${ }^{1}$ Leibniz-Institute of Vegetables and Ornamental Crops Großbeeren/Erfurt e.V, Großbeeren, Germany \\ ${ }^{2}$ Institute of Nutritional Science, University of Potsdam, Nuthetal, Germany \\ ${ }^{3}$ South China Botanical Garden, Chinese Academy of Sciences, Guangzhou, China \\ ${ }^{4}$ Shizuoka Prefectural Research Institute of Agriculture and Forestry Tea Research Center, Shizuoka, Japan \\ ${ }^{5}$ Faculty of Agriculture, Shizuoka University, Shizuoka, Japan \\ ${ }^{6}$ Graduate School of Engineering, Shizuoka University, Hamamatsu, Japan \\ ${ }^{7}$ Graduate School of Science and Technology, Shizuoka University, Shizuoka, Japan \\ Email: ${ }^{*}$ acnwata@ipc.shizuoka.ac.jpa
}

Received 22 April 2014; revised 2 June 2014; accepted 19 June 2014

Copyright (C) 2014 by authors and Scientific Research Publishing Inc.

This work is licensed under the Creative Commons Attribution International License (CC BY).

http://creativecommons.org/licenses/by/4.0/

(c) (i) Open Access

\section{Abstract}

Tea is one of the most consumed beverages in the world and its quality is influenced by geographical origin and production methods. This study focuses on the volatile aroma components of 38 tea products from China, Japan, Indonesia, Sri-Lanka, and Chinese Taipei; among them 7 green teas, 13 oolong teas, and 18 black teas. The volatiles were extracted from the infusions using PorapakQ-resin, concentrated, and analyzed by gas chromatography-mass spectrometry. The components were identified by authentic reference compounds or preliminary based on their mass spectra. Different manufacturing processes yield different blends of aroma compounds. In general, the contents of total volatiles, aliphatics, aromatics, and terpenoids increased with the fermentation degree, whereas jasmine lactone and indole were the highest in oolong teas. Some particular manufacturing processes, for example, the use of tea leaves infested by the tea green leafhopper, lead to higher contents of volatiles in final products as in Oriental Beauty oolong tea. The relative peak areas determined for $\mathbf{8 2}$ volatiles were the basis for the statistical analysis and highlight the potential of multivariate analysis to distinguish tea samples of different categories.

\section{Keywords}

Aroma, Camellia sinensis, Fermentation, Clustering Analysis, Tea

\footnotetext{
${ }^{*}$ Corresponding author.
}

How to cite this paper: Baldermann, S., Yang, Z.Y., Katsuno, T., Tu, V.A., Mase, N., Nakamura, Y. and Watanabe, N. (2014) Discrimination of Green, Oolong, and Black Teas by GC-MS Analysis of Characteristic Volatile Flavor Compounds. American Journal of Analytical Chemistry, 5, 620-632. http://dx.doi.org/10.4236/ajac.2014.59070 


\section{Introduction}

Tea (Camellia sinensis) is a plant commercially grown for beverage production. Until now, more than 300 different kinds of tea are produced from the leaves of $C$. sinensis by different fermentation processes. Commonly teas are classified based on the manufacturing process and can be divided up into six major families, including non-fermented green tea, slightly fermented white tea, semi-fermented oolong tea, fully fermented black tea, post fermented yellow tea, and dark (red) tea (Figure 1). White tea, yellow tea, and dark tea are characteristic Chinese teas and particularly popular in China, whereas green tea, oolong tea, and black tea are well-known all around the world and in particular focus of this study. From a viewpoint of tea quality evaluation, tea aroma is one of the main sensory properties which are decisive in selection, acceptance and ingestion of final tea products. Formation of tea aroma is influenced by different manufacturing processes. For example, the particular manufacturing process of oolong tea gives a unique floral, fruity, and jasmine-like aroma [1] [2]. The aroma compounds of tea such as green tea, oolong tea and black tea have been individually investigated by gas chromatography-mass spectrometry (GC-MS) or GC-olfactometry (GC-O) [3]-[7]. In addition various approaches were employed for the discrimination of teas from different geographical origins such as high performance liquid chromatography [8], capillary electrophoresis [9] or electronic nose [10].

In our study, we collected more than 38 kinds of tea products including green teas, oolong teas, and black teas from different production areas, investigated their volatile compounds to study the different manufacturing processes on the tea aroma profiles as well as relationships between particular processes and tea aroma compounds. We aimed to develop a fast method to determine the origin based on profiling of volatiles by GC-MS and statistical analysis.

\section{Experimental}

\subsection{Tea Samples}

In total 38 tea products of high grade were obtained from tea exporters or research centers from China, Japan, Indonesia, Sri-Lanka, and Chinese Taipei; among them 7 green teas, 13 oolong teas, and 18 black teas. Supplementary Table S1 summarizes names, origin, and other characteristics of tea samples investigated in this study.

\subsection{Analysis of Tea Volatile Compounds by GC-MS}

Deionized hot water $\left(40 \mathrm{~mL}, 80^{\circ} \mathrm{C}\right)$ was added to $2 \mathrm{~g}$ of the tea product. After 5 min the leaves were removed by nylon filter and small residues by centrifugation at $3000 \mathrm{~g}$ for $10 \mathrm{~min} .25 \mathrm{~mL}$ of the supernatant were passed through a $2 \mathrm{~mL}$ PorapakQ-cartridge, conditioned with diethyl ether $(5 \times 4 \mathrm{~mL})$, methanol $(4 \mathrm{~mL})$, and water $(4 \mathrm{~mL})$. The cartridge was washed with $3 \mathrm{~mL}$ of deionized water prior elution of the volatile compounds with $3 \mathrm{~mL}$

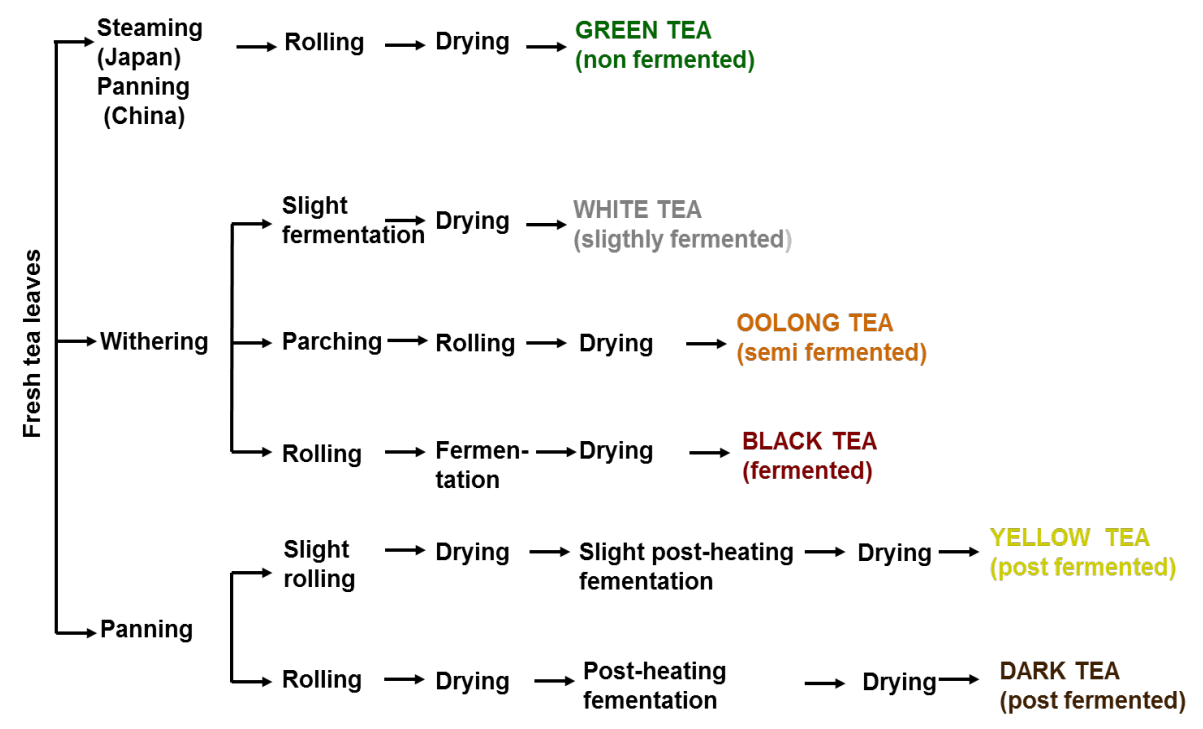

Figure 1. Simplified production processes of various kinds of tea products. 
iso-pentane: diethyl ether (1:1, v/v). For relative quantification ethyl decanoate (54.1 pmol) was added as an internal standard and the organic layer dried over anhydrous sodium sulfate. The eluate was concentrated to $100 \mu \mathrm{L}$ in a stream of nitrogen. One $\mu \mathrm{L}$ concentrate was subjected to GC-MS analysis for identification and relative quantification of the volatiles. Splitless injection mode was used with a splitless time of 1 min and an injector temperature of $230^{\circ} \mathrm{C}$. Helium was the carrier gas with a velocity of $1.7 \mathrm{~mL} \cdot \mathrm{min}^{-1}$. The GC was equipped with a capillary SUPELCOWAX ${ }^{\mathrm{TM}} 10$ column $(30 \mathrm{~m} \times 0.25 \mathrm{~mm}$ i.d. and $0.25 \mu \mathrm{m}$ film thickness). The GC oven was maintained at $50^{\circ} \mathrm{C}$ for $3 \mathrm{~min}$ and then heated at a rate of $3^{\circ} \mathrm{C} \cdot \mathrm{min}^{-1}$ to $150^{\circ} \mathrm{C}$ followed by a heating rate of $20^{\circ} \mathrm{C} \mathrm{min}^{-1}$ to $240^{\circ} \mathrm{C}$ and kept at this temperature for $20 \mathrm{~min}$. The mass scan range was $\mathrm{m} / \mathrm{z} 50-300$ and the electric potential was set to $\mathrm{EI} 70 \mathrm{eV}$.

\subsection{Statistical Analysis}

Peak areas of volatiles detected between 4.5 and 58 min were extracted from the mass chromatograms and relative concentrations calculated using the internal standard ethyl decanoate. Clustering analysis by STATISTICA (StatSoft, Inc. (2013). data analysis software system, version 12. www.statsoft.com ) was employed for the visualization of the datasets. In this work, 82 volatiles in 38 teas were taken into account. Components identified solely in one tea were excluded from the statistical analysis.

\section{Results and Discussion}

Compositions of tea products have been investigated by many researchers. Phenolic constituents, organic acids, caffeine, and volatiles are key chemical components defining taste and flavor.

Flavor as a key parameter for tea quality is greatly influenced by brewing temperature, brewing time, extraction methods, and many other factors.

In this study we compared the volatile profiles of green teas, oolong teas, and black teas using equal extraction conditions for all samples. Therefore, our results represent differences mainly caused by manufacturing processes (Figure 1) and variation of raw materials including the geographic origin.

Based on the observations on volatile compounds of 38 tea samples (Table S1) including green teas (nonfermented), oolong teas (semi-fermented), and black teas (fully fermented), the contents of total volatiles, aliphatics, aromatics, and terpenoids increased with the fermentation degree (Figure 2(a) \& Figure 2(b)).

Clustering analysis was performed to discriminate between tea samples of different categories. As a result of the statistical analysis we could obtain clusters of the different tea categories and distinguish samples according to the origin (Figure 3).

In general, the aroma profiles of black teas and oolong teas are more complex than the ones of green teas. In green tea fewer volatiles can be found and among the 200 volatiles about 30 compounds essentially contribute to the typical green tea aroma [5] [6]. Besides short chained alcohols and aldehydes, geraniol, linalool, 2-phenylethanol, benzyl alcohol, indole, and coumarin lead to green tea aroma. In black tea infusions about 600 constituents have been identified and 41 compounds importantly contribute to the aroma of black tea infusions [11].

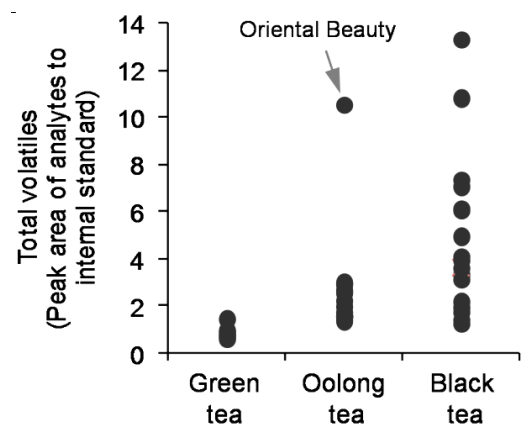

(a)

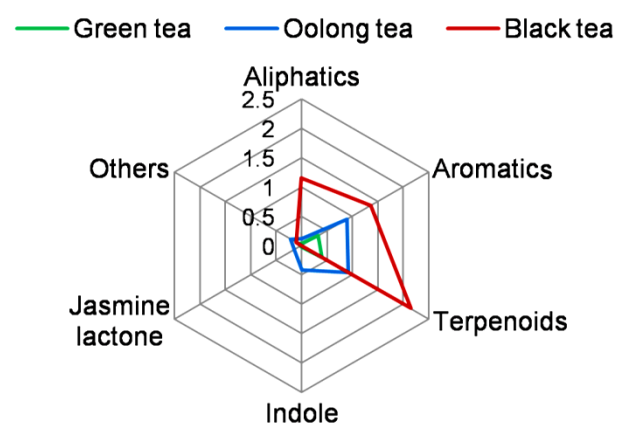

(b)

Figure 2. Comparisons of total volatile contents (a) and classified volatiles of green teas, oolong teas, and black teas (Tables S1-S4). Total volatiles contents were represented as area ratio of volatiles to internal standard (ethyl decanoate); (b) The average values of classified volatiles of green teas, oolong teas and black teas. 


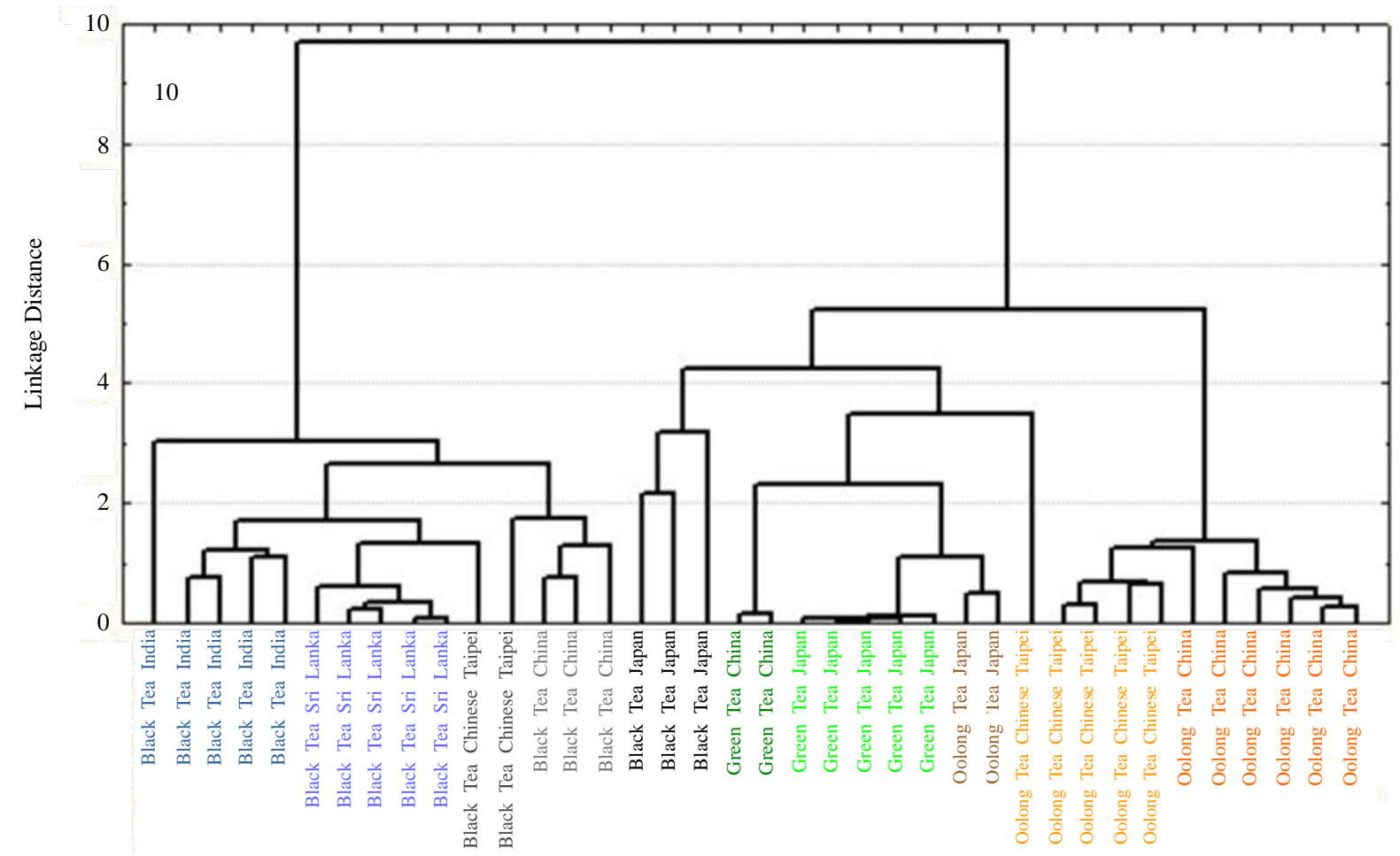

Figure 3. Clustering analyses of the relative peak areas (TIC, mass range $\mathrm{m} / \mathrm{z} 50$ - 300, 82 compounds) of 38 teas (Table S1).

Several of these important aroma compounds have been found in all kinds of black tea, among them Z-3-hexen1-ol, linalool and its oxides, geraniol, and 2-phenylethanol contributing to the green, citrus-like, rose-like and honey-like notes, respectively. Linalool and its oxides, benzylalcohol, and 2-phenylethanol were detected as volatiles in all oolong teas. Although contents of most volatiles in black teas are higher than oolong teas and green teas, jasmine lactone and indole were the highest in oolong teas. Both volatiles possess jasmine-like floral and fruity fragrances and importantly contribute to oolong tea aroma [12]. This result is identical with previous reports [13]. Methyl salicylate, previously described as characteristic aroma component of oolong tea, has been only found in some teas of Chinese Taipei (Table S1 No. 7, 8, and 10) or Japanese (Table S1 12 and 13 ) origin [14].

Many aroma compounds occur as glycosidic precursors in fresh tea leaves [15], and they are hydrolyzed by endogenous glycosidases during the manufacturing processes of withering, rolling, and fermentation [1] [2] [16]. During the production of green teas, plucked fresh tea leaves are steamed or pan-fired to inactivate activity of enzymes including glycosidases related to hydrolysis of glycosidically bound volatiles. In contrast, endogenous enzymatic reactions occur in the several processes of oolong teas and black teas, which results in the accumulation of aroma compounds such as aliphatics, aromatics, and terpenoids in final products (Figure 2(b)). During the rolling process of black tea, the structure of the leaf cells is disrupted and the contents of the cells are completely mixed, so the hydrolysis of glycosidically bound aroma precursors plays a major role in the formation of black tea aroma [1]. In contrast, in the processes of oolong teas, one or more biosynthetic pathways, such as the formation of jasmine lactone and indole, might take priority over the hydrolysis of glycosidically bound aroma precursors, although the mechanism for the formation of the two volatiles has not yet been clarified [2]. In the sensory evaluation, oolong teas are generally considered to possess more pleasant flavor than black teas, although these contain higher contents of tea volatiles. This suggests that some characteristic aroma compounds are produced during the manufacturing process of oolong tea. Alive time of tea leaves in the process of oolong tea is much longer time than that in the process of black tea [17]. Therefore, tea leaves during oolong tea manufacturing process are exposed longer to various stresses including plucking (wounding), solar withering (drought, heat, and UV radiation), indoor withering (drought), and turn over (wounding) [18]. In addition, biotic stress 
such as insect infestation is involved in the oolong tea manufacturing. A typical example is a famous Formosa oolong tea (Oriental Beauty) that has a unique aroma like ripe fruits and honey. It contains the highest content of total volatiles among the oolong teas (Figure 2(a)). This may be due to the formation of unique volatiles in tea leaves under insect attack [18]. In the Oriental Beauty manufacturing process, one of the most characteristic factors is the use of tea leaves infested by the tea green leafhopper. It has been reported that formations of many volatiles in tea leaves can be induced by insect attack [19] [20]. Taken together, the aroma formation in tea leaves during the manufacturing process may be the result of defense responses of tea leaves against various stresses. Hence, more detailed molecular studies on stress-induced volatile formation during the tea manufacturing process will provide an important basis for improvement of flavor quality of other teas.

\section{Conclusion}

In this study, a comprehensive comparison of aroma profiles of green teas, oolong teas, and black teas collected from different production areas was performed. Fermentation intensity influences the quantity of most tea volatiles during the manufacturing process. Besides fermentation, some particular stress-related manufacturing processes, for example during the production of oolong tea, result in the formation of some characteristic tea volatiles such as jasmine lactone or indole. These results will contribute to our further understanding of the effects of different manufacturing processes on tea aroma profile, and provide essential information for further development of aroma-enriched tea products. Moreover, this study could form the basis for authenticity studies of tea products.

\section{Acknowledgements}

This study was supported by a project "From Shizuoka to the world: Research and development of next-generation bottled tea drinks and tea extracts" of Shizuoka Prefecture and Shizuoka City Collaboration of Regional Entities for the Advancement of Technological Excellence, Japan Science and Technology Agency (JST).

\section{References}

[1] Wang, D.M., Kurasawa, E., Yamaguchi, Y., Kubota, K. and Kobayashi, A. (2001) Analysis of Glycosidically Bound Aroma Precursors in Tea Leaves. 2. Changes in Glycoside Contents and Glycosidase Activities in Tea Leaves during the Black Tea Manufacturing Process. Journal of Agricultural and Food Chemistry, 49, 1900-1903. http://dx.doi.org/10.1021/jf001077+

[2] Wang, D.M., Kubota, K., Kobayashi, A. and Juan, I.M. (2001) Analysis of Glycosidically Bound Aroma Precursors in Tea Leaves. 3. Changes in Glycoside Contents during the Oolong Tea Manufacturing Process. Journal of Agricultural and Food Chemistry, 49, 5391-5396. http://dx.doi.org/10.1021/jf010235+

[3] Shimoda, M., Shigematsu, H., Shiratsuchi, H. and Osajima, Y. (1995) Comparison of the Odor Concentrates by SDE and Adsorptive Column Method from Green Tea Infusion. Journal of Agricultural and Food Chemistry, 43, 1616-1620. http://dx.doi.org/10.1021/jf00054a037

[4] Kawakami, M., Ganguly, S.N., Banerjee, J. and Kobayashi, A. (1995) Aroma Composition of Oolong Tea and Black Tea by Brewed Extraction Method and Characterizing Compounds of Darjeeling Tea Aroma. Journal of Agricultural and Food Chemistry, 43, 200-207. http://dx.doi.org/10.1021/jf00049a037

[5] Kumazawa, K. and Masuda, H. (1999) Identification of Potent Odorants in Japanese Green Tea (Sen-Cha). Journal of Agricultural and Food Chemistry, 47, 5169-5172. http://dx.doi.org/10.1021/jf9906782

[6] Kumazawa, K. and Masuda, H. (2002) Identification of Potent Odorants in Different Green Tea Varieties Using Flavor Dilution Technique. Journal of Agricultural and Food Chemistry, 50, 5660-5663. http://dx.doi.org/10.1021/jf020498j

[7] Ye, N., Zhang, L. and Gu, X. (2012) Discrimination of Green Teas from Different Geographical Origins by Using HSSPME/GC-MS and Pattern Recognition Methods. Food Analytical Methods, 5, 856-860. http://dx.doi.org/10.1007/s12161-011-9319-9

[8] Fernández, P., Pablos, F., Martín, M.J. and González, A.G. (2002) Study of Catechin and Xanthine Tea Profiles as Geographical Tracers. Journal of Agricultural and Food Chemistry, 50, 1833-1839.

[9] Ye, N.S., Zhang, L. and Gu, X. (2011) Classification of Maojian Teas from Different Geographical Origins by Micellar Electrokinetic Chromatography and Pattern Recognition Techniques. Analytical Sciences, 27, 765-769.

[10] Kovács, Z., Dalmadi, I., Lukács, L., Sipos, L., Szántai-Kőhegyi, K., Kókai, Z. and Fekete, A. (2010) Geographical Origin Identification of Pure Sri Lanka Tea Infusions with Electronic Nose, Electronic Tongue and Sensory Profile 
Analysis. Journal of Chemometrics, 24, 121-130.

[11] Schuh, C. and Schieberle, P. (2006) Characterization of the Key Aroma Compounds in Beverage Prepared from Darjeeling Black Tea: Quantitative Differences between Tea Leaves and Infusion. Journal of Agricultural and Food Chemistry, 54, 916-924. http://dx.doi.org/10.1021/jf052495n

[12] Yamanishi, T., Kosuge, M., Tokitomo, Y. and Maeda, R. (1980) Flavor Constituents of Pouchong Tea and a Comparison of the Aroma Pattern with Jasmine Tea. Agricultural and Biological Chemistry, 44, 2139-2142. http://dx.doi.org/10.1271/bbb1961.44.2139

[13] Zhang, L., Zeng, Z., Zhao, C., Kong, H., Lu, X. and Xu, G. (2013) A Comparative Study of Volatile Components in Green, Oolong and Black Teas by Using Comprehensive Two-Dimensional Gaschromatography-Time-of-Flight Mass Spectrometry and Multivariate Data Analysis. Journal of Chromatography A, 1313, 245-252. http://dx.doi.org/10.1016/j.chroma.2013.06.022

[14] Chen, Y.L., Duan, J., Jiang, Y.M., Shi, J., Peng, L., Xue, S. and Kakuda, Y. (2010) Production, Quality, and Biological Effects of Oolong Tea (Camellia sinensis). Food Reviews International, 27, 1-15. http://dx.doi.org/10.1080/87559129.2010.518294

[15] Wang, D.M., Yoshimura, T., Kubota, K. and Kobayashi, A. (2000) Analysis of Glycosidically Bound Aroma Precursors in Tea Leaves. 1. Qualitative and Quantitative Analyses of Glycosides with Aglycons as Aroma Compounds. Journal of Agricultural and Food Chemistry, 48, 5411-5418. http://dx.doi.org/10.1021/jf000443m

[16] Kinoshita, T., Hirata, S., Yang, Z.Y., Baldermann, S., Kitayama, E., Matsumoto, S., Suzuki, M., Fleischmann, P., Winterhalter, P. and Watanabe, N. (2010) Formation of Damascenone Derived from Glycosidically Bound Precursors in Green Tea Infusions. Food Chemistry, 123, 601-606. http://dx.doi.org/10.1016/j.foodchem.2010.04.077

[17] Sakata, K., Mizutani, M., Cho, J.Y., Kinoshita, T. and Shimizu, B. (2008) Improvement of Flavour Quality of Black Tea Using Molecular Basis of Aroma Formation in Oolong Tea. In: Jain, N.K., Rahamn, F. and Baker, P., Eds., Economic Crisis in Tea Industry, Studium Press LLC, Houston, 212-225.

[18] Cho, Y.M., Mizutani, M., Shimizu, B., Kinoshita, T., Ogura, M., Tokoro, K., Lin, M.L. and Sakata, K. (2007) Chemical Profiling and Gene Expression Profiling during the Manufacturing Process of Taiwan Oolong Tea "Oriental Beauty”. Bioscience Biotechnology and Biochemistry, 71, 1476-1486. http://dx.doi.org/10.1271/bbb.60708

[19] Han, B.Y. and Chen, Z.M. (2002) Composition of the Volatiles from Intact and Mechanically Pierced Tea Aphid-Tea Shoot Complexes and Their Attraction to Natural Enemies of the Tea Aphid. Journal of Agricultural and Food Chemistry, 50, 2571-2575. http://dx.doi.org/10.1021/jf010681x

[20] Dong, F., Yang, Z.Y., Baldermann, S., Sato, Y., Asai, T. and Watanabe, N. (2011) Herbivore-Induced Volatiles from Tea (Camellia sinensis) Plants and Their Involvement in Intraplant Communication and Changes in Endogenous NonVolatile Metabolites. Journal of Agricultural and Food Chemistry, 59, 13131-13135. http://dx.doi.org/10.1021/jf203396a 


\section{Supplement}

Table S1. Classification and geographical origin of tea samples.

\begin{tabular}{|c|c|c|c|c|}
\hline No. & Commercial name & Tea type & Origin & Others characteristics \\
\hline 1 & Dahongpao & Oolong & China & Roasted \\
\hline 2 & Buzhichun & Oolong & China & Roasted \\
\hline 3 & Rougui & Oolong & China & Roasted \\
\hline 4 & Yunxiang 999 & Oolong & China & Pellet type \\
\hline 5 & Tieguanyin AAA & Oolong & China & Pellet type \\
\hline 6 & Nashiyama & Oolong & Chinese Taipei & Roast, high mountain, pellet type \\
\hline 7 & Nashiyama & Oolong & Chinese Taipei & High mountain, pellet type \\
\hline 8 & Kouzancha & Oolong & Chinese Taipei & High mountain, organic, pellet type \\
\hline 9 & Bunzanhoushycha & Oolong & Chinese Taipei & \\
\hline 10 & Oriental Beauty & Oolong & Chinese Taipei & \\
\hline 11 & Shikicha & Oolong & Chinese Taipei & Pellet type \\
\hline 12 & Shizu 7132 & Oolong & Japan & \\
\hline 13 & Benifuuki & Oolong & Japan & \\
\hline 14 & Zhuangyuanhong & Green & China & \\
\hline 15 & Baichazu & Green & China & \\
\hline 16 & Yabukita & Green & Japan & \\
\hline 17 & Kousyun & Green & Japan & \\
\hline 18 & Tsuyuhikari & Green & Japan & \\
\hline 19 & Okuhikari & Green & Japan & \\
\hline 20 & Fujiedakaori & Green & Japan & \\
\hline 21 & Benifuuki & Black & Japan & Plucked by hand \\
\hline 22 & Yabukita & Black & Japan & Organic \\
\hline 23 & Benifuuki & Black & Japan & \\
\hline 24 & Lapsang Souchong & Black & China & \\
\hline 25 & Bainianlaoshu & Black & China & \\
\hline 26 & Jinjunmei & Black & China & \\
\hline 27 & Mitsukoukoucha & Black & Chinese Taipei & \\
\hline 28 & Fbobf Extra Special New Tokutou & Black & Chinese Taipei & \\
\hline 29 & Vithanakande Tea Factory & Black & Sri Lanka & \\
\hline 30 & Fbobf Special New Vithanakande Tea Factory & Black & Sri Lanka & \\
\hline 31 & Ruhunu B.O.P.I & Black & Sri Lanka & \\
\hline 32 & A Fine Uva Tea & Black & Sri Lanka & \\
\hline 33 & A Fine Dimbula tea & Black & Sri Lanka & \\
\hline 34 & Rubi-Bio-Organic & Black & India & Organic \\
\hline 35 & Puttabong & Black & India & 2nd flush \\
\hline 36 & 2010 Thurbo & Black & India & 2nd flush \\
\hline 37 & Jungpana & Black & India & 1st flush \\
\hline 38 & Rohini & Black & India & 2nd flush \\
\hline
\end{tabular}


Table S2. Volatiles determined in oolong tea (relative concentrations, bold-indentified by authentic reference material).

\begin{tabular}{|c|c|c|c|c|c|c|c|c|c|c|c|c|c|c|}
\hline & & \multicolumn{13}{|c|}{ Oolong tea } \\
\hline & & TEA 1 & TEA 2 & TEA 3 & TEA 4 & TEA 5 & TEA 6 & TEA 7 & TEA 8 & TEA 9 & TEA 10 & TEA 11 & TEA 12 & TEA 13 \\
\hline 1 & Hexanal & 0 & 0.012507 & 0 & 0 & 0 & 0 & 0 & 0 & 0 & 0.03191 & 0 & 0 & 0.020512 \\
\hline 2 & Undecan & 0 & 0 & 0 & 0 & 0 & 0 & 0 & 0 & 0 & 0 & 0 & 0 & 0.012185 \\
\hline 3 & $\begin{array}{l}\text { Methylpentenone } \\
\text { derivative }\end{array}$ & 0 & 0.005117 & 0 & 0 & 0 & 0.010494 & 0 & 0 & 0 & 0 & 0 & 0 & 0 \\
\hline 4 & 1-Penten-3-ol & 0.037501 & 0.025021 & 0 & 0.017292 & 0.018767 & 0 & 0.011003 & 0.004343 & 0 & 0 & 0 & 0.008701 & 0.032568 \\
\hline 5 & unknown 1 & 0.039955 & 0.026682 & 0.09611 & 0 & 0 & 0 & 0 & 0 & 0 & 0.08007 & 0 & 0 & 0 \\
\hline 6 & $\begin{array}{l}\text { Methylbutanol } \\
\text { derivative }\end{array}$ & 0 & 0 & 0 & 0.003325 & 0 & 0 & 0.004398 & 0.002038 & 0 & 0.03211 & 0.002753 & 0.005442 & 0 \\
\hline 7 & 2-Hexenal,(E)- & 0 & 0 & 0 & 0 & 0 & 0 & 0 & 0 & 0 & 0 & 0 & 0 & 0 \\
\hline 8 & 2,5-Dimethylpyrazine & 0.009007 & 0.009209 & 0.01504 & 0 & 0 & 0 & 0 & 0 & 0 & 0 & 0 & 0 & 0 \\
\hline 9 & $\begin{array}{l}\text { Diexthylbenzene } \\
\text { derivative }\end{array}$ & 0 & 0 & 0 & 0 & 0 & 0 & 0 & 0 & 0.04951 & 0.0636 & 0.043865 & 0.053425 & 0.011821 \\
\hline 10 & $\begin{array}{l}\text { 2,5-Dimethyl } \\
\text { pyrazine }\end{array}$ & 0.009791 & 0.008878 & 0.01312 & 0 & 0 & 0 & 0 & 0 & 0 & 0 & 0 & 0 & 0 \\
\hline 11 & 2-Penten-1-ol & 0.011078 & 0.009895 & 0 & 0 & 0.005613 & 0.033937 & 0 & 0 & 0 & 0.0218 & 0 & 0.005439 & 0.011716 \\
\hline 12 & Benzene,1,3-diethyl- & 0 & 0 & 0 & 0 & 0 & 0 & 0 & 0 & 0.003964 & 0.00578 & 0.004516 & 0 & 0 \\
\hline 13 & 2-Ethyl pyrazine & 0.006935 & 0.006448 & 0 & 0 & 0 & 0 & 0 & 0 & 0 & 0 & 0 & 0 & 0 \\
\hline 14 & 1-Hexanol & 0.003793 & 0.002463 & 0 & 0 & 0 & 0 & 0 & 0 & 0 & 0.06138 & 0 & 0 & 0.00081 \\
\hline 15 & 3-Hexen-1-ol & 0 & 0 & 0 & 0.004862 & 0 & 0.011102 & 0.008905 & 0 & 0 & 0.14125 & 0.004808 & 0.012967 & 0.017699 \\
\hline 16 & 2-Hexen-1-ol, (E)- & 0 & 0 & 0 & 0 & 0 & 0 & 0 & 0 & 0 & 0.02006 & 0 & 0 & 0 \\
\hline 17 & Pentylalcohol & 0.002031 & 0.002038 & 0 & 0.004423 & 0.002909 & 0.005995 & 0.003713 & 0.001498 & 0.001608 & 0 & 0.001734 & 0.002299 & 0 \\
\hline 18 & Linalool oxide I & 0.278193 & 0.453098 & 0.22692 & 0.360846 & 0.340765 & 0.552482 & 0.630453 & 0.277981 & 0.282035 & 1.91485 & 0.307824 & 0.427458 & 0.364645 \\
\hline 19 & 2-Furancarboxaldehyde & 0.043668 & 0.063263 & 0.12185 & 0 & 0.045555 & 0.03744 & 0 & 0 & 0 & 0 & 0 & 0 & 0 \\
\hline 20 & 2,4-Heptadienal & 0 & 0 & 0 & 0 & 0 & 0 & 0 & 0 & 0 & 0 & 0 & 0 & 0 \\
\hline 21 & Furan,2,5-dimethyl & 0 & 0 & 0 & 0 & 0 & 0 & 0 & 0 & 0 & 0 & 0 & 0 & 0 \\
\hline 22 & Linalool oxide II & 0.05165 & 0.022172 & 0.09671 & 0 & 0 & 0.027484 & 0.017367 & 0.019146 & 0 & 1.28653 & 0 & 0.0593 & 0.066841 \\
\hline 23 & Furan-2-propyl & 0 & 0.005314 & 0 & 0 & 0 & 0 & 0 & 0 & 0 & 0.0155 & 0 & 0 & 0 \\
\hline 24 & $\begin{array}{l}\text { Ethanone, } \\
\text { 1-(2-furanyl)- }\end{array}$ & 0.01129 & 0.014791 & 0.02296 & 0 & 0 & 0.004912 & 0 & 0 & 0 & 0 & 0 & 0 & 0 \\
\hline 25 & Benzenaldehyde & 0 & 0 & 0 & 0.016045 & 0 & 0 & 0.002918 & 0.000566 & 0.006439 & 0.13214 & 0 & 0.00144 & 0.010979 \\
\hline 26 & Linalool & 0.00325 & 0.006247 & 0 & 0.001932 & 0 & 0.009178 & 0.023261 & 0.023418 & 0.008339 & 0.10796 & 0.006303 & 0.015558 & 0.029783 \\
\hline 27 & $\begin{array}{l}\text { 2-Furancarboxaldehyde, } \\
\text { 5-methyl- }\end{array}$ & 0.024404 & 0.024827 & 0.08507 & 0 & 0.003857 & 0.009808 & 0 & 0 & 0 & 0 & 0 & 0 & 0 \\
\hline 28 & $\begin{array}{l}\text { 2-Heptanone, } \\
\text { 5-methyl- }\end{array}$ & 0.004989 & 0.0056 & 0 & 0 & 0 & 0 & 0 & 0 & 0 & 0 & 0 & 0 & 0 \\
\hline 29 & $\begin{array}{c}\text { 1H-pyrrole- } \\
\text { 2carboxaldehyde, }\end{array}$ & 0.063601 & 0.042581 & 0 & 0 & 0.006086 & 0.060033 & 0.003038 & 0 & 0 & 0 & 0 & 0 & 0 \\
\hline 30 & $\begin{array}{l}\text { 1,5,7-Octatrien-3-ol, } \\
\text { 3,7-dimethyl- }\end{array}$ & 0.026713 & 0.048355 & 0.03544 & 0 & 0.031357 & 0.141985 & 0.014979 & 0.003328 & 0.001759 & 0.45281 & 0.007886 & 0.002776 & 0.002531 \\
\hline 31 & $\begin{array}{l}\text { Benzenamine, } \\
\text { 2-methoxy-5-methyl }\end{array}$ & 0.006261 & 0.007924 & 0 & 0 & 0 & 0.00434 & 0 & 0 & 0 & 0 & 0 & 0 & 0 \\
\hline 32 & 2-Furanmethanol & 0.01308 & 0.019734 & 0 & 0 & 0.008345 & 0.0227 & 0 & 0 & 0 & 0 & 0 & 0 & 0 \\
\hline 33 & $\begin{array}{l}\text { 2(3H)-furanone, } \\
\text { 5-ethyldihydro }\end{array}$ & 0 & 0 & 0 & 0 & 0 & 0 & 0 & 0 & 0 & 0 & 0.002678 & 0 & 0 \\
\hline 34 & $\begin{array}{l}\text { 2(3H)-furanone, } \\
\text { 5-ethyldihydro }\end{array}$ & 0.009178 & 0.021197 & 0.01589 & 0.004729 & 0.02004 & 0.035016 & 0.020629 & 0.004609 & 0.003329 & 0.00515 & 0 & 0 & 0 \\
\hline 35 & $\begin{array}{c}\text { 4-Ethyl } \\
\text { benzaldehyde }\end{array}$ & 0 & 0 & 0 & 0.013753 & 0 & 0 & 0 & 0 & 0.043394 & 0.04822 & 0.042805 & 0.058109 & 0 \\
\hline 37 & $\begin{array}{l}\text { Butanoic acid, } \\
\text { 2-methyl }\end{array}$ & 0 & 0 & 0 & 0 & 0 & 0 & 0 & 0 & 0 & 0 & 0 & 0 & 0 \\
\hline 38 & $\begin{array}{l}\text { 1-Isopropyl-2-methoxy } \\
\text {-4-methylbenzene }\end{array}$ & 0 & 0 & 0 & 0 & 0 & 0 & 0 & 0 & 0 & 0 & 0 & 0 & 0.062645 \\
\hline
\end{tabular}




\section{Continued}

\begin{tabular}{|c|c|c|c|c|c|c|c|c|c|c|c|c|c|c|}
\hline 39 & Linalool oxide III & 0.038904 & 0.043245 & 0.09993 & 0.008465 & 0.016364 & 0.026601 & 0.038624 & 0.022874 & 0.011986 & 0.94901 & 0.014859 & 0.051462 & 0.051958 \\
\hline 40 & $\begin{array}{l}\text { Benzenamine, } \\
\text { 4-ethoxy- }\end{array}$ & 0.004993 & 0.010665 & 0.00482 & 0 & 0 & 0.012728 & 0.020429 & 0.013741 & 0.002369 & 0.61736 & 0.003748 & 0.118391 & 0.109938 \\
\hline 41 & Linalool oxide IV & 0.025054 & 0.002292 & 0 & 0 & 0 & 0.002881 & 0.006268 & 0.004644 & 0 & 0.08887 & 0.000547 & 0.002685 & 0.003629 \\
\hline 42 & Methyl salicylate & 0.004201 & 0 & 0 & 0 & 0 & 0 & 0 & 0 & 0 & 0 & 0 & 0 & 0 \\
\hline 43 & Nerol & 0 & 0 & 0 & 0 & 0 & 0 & 0 & 0 & 0 & 0.01623 & 0 & 0 & 0 \\
\hline 44 & $\begin{array}{l}\text { Benzoic acid,4- } \\
\text { ethyl, methyl ester }\end{array}$ & 0 & 0 & 0 & 0.0047 & 0 & 0 & 0 & 0 & 0 & 0 & 0 & 0 & 0.083049 \\
\hline 45 & $\begin{array}{l}\text { 3,4-Dimethyl } \\
\text { acetophenone }\end{array}$ & 0 & 0 & 0 & 0.040009 & 0 & 0 & 0 & 0 & 0.079507 & 0.08847 & 0.078326 & 0.103054 & 0.037573 \\
\hline 46 & $\begin{array}{c}\text { Benzoic acid, } \\
\text { 4-formyl-,methylester }\end{array}$ & 0 & 0 & 0 & 0 & 0 & 0 & 0 & 0 & 0 & 0 & 0 & 0 & 0 \\
\hline 47 & O-Diacetylbenzene & 0 & 0 & 0 & 0 & 0 & 0 & 0 & 0 & 0 & 0 & 0 & 0 & 0 \\
\hline 48 & Geraniol & 0.006842 & 0.01203 & 0 & 0 & 0 & 0.025202 & 0.119208 & 0.049504 & 0 & 0.38022 & 0.011039 & 0 & 0.071248 \\
\hline 49 & 4-Ethyl acetophenone & 0 & 0 & 0 & 0.033925 & 0 & 0 & 0 & 0 & 0.055803 & 0 & 0.053107 & 0.003044 & 0.054907 \\
\hline 50 & Benzyl alcohol & 0.098772 & 0.087601 & 0.17541 & 0.066293 & 0.041059 & 0.113813 & 0.08896 & 0.036744 & 0.028317 & 1.71382 & 0.036558 & 0.044358 & 0.139831 \\
\hline 51 & 2-Phenylethanol & 0.144479 & 0.128073 & 0.27446 & 0.626597 & 0.368093 & 0.078673 & 0.086613 & 0.045294 & 0.160206 & 1.36397 & 0.08072 & 0.064703 & 0.154794 \\
\hline 53 & Methyl cinamate & 0 & 0 & 0 & 0 & 0 & 0 & 0 & 0 & 0 & 0 & 0 & 0 & 0 \\
\hline 54 & Hexanoic acid & 0 & 0 & 0 & 0 & 0 & 0 & 0 & 0 & 0 & 0.30249 & 0 & 0 & 0 \\
\hline 55 & Benzeneacetonitrile & 0.048178 & 0.070401 & 0.11509 & 0.063322 & 0.056501 & 0.03622 & 0.027845 & 0.008752 & 0.078826 & 0 & 0.018449 & 0 & 0 \\
\hline 56 & Jasmone & 0 & 0 & 0 & 0 & 0 & 0 & 0.022476 & 0.013458 & 0.005838 & 0 & 0 & 0.004618 & 0 \\
\hline 57 & $\begin{array}{l}\text { Butanoic acid, } \\
\text { 3-hexenyl ester }\end{array}$ & 0 & 0 & 0 & 0.001616 & 0 & 0 & 0 & 0 & 0 & 0 & 0 & 0 & 0 \\
\hline 58 & $\begin{array}{l}\text { 3,7-Octadien-2, } \\
\text { 6-diol,2,6-dimethyl- }\end{array}$ & 0 & 0 & 0 & 0 & 0.002275 & 0 & 0.013942 & 0.005489 & 0 & 0.16256 & 0.002639 & 0.007096 & 0 \\
\hline 59 & $\begin{array}{c}\text { Ethanone, } \\
\text { 1-(1H-pyrrol-2-yl)- }\end{array}$ & 0.065064 & 0.120217 & 0.12849 & 0 & 0.017023 & 0.081787 & 0.006378 & 0 & 0 & 0 & 0 & 0 & 0 \\
\hline 60 & $\begin{array}{l}\text { 4-(1-hydroxyethyl) } \\
\text { benzaldehyde }\end{array}$ & 0 & 0 & 0 & 0.0153 & 0 & 0 & 0 & 0 & 0.178687 & 0 & 0 & 0 & 0 \\
\hline 61 & $\begin{array}{l}\text { 4-Hydroxy-3- } \\
\text { methylacetophenone }\end{array}$ & 0 & 0 & 0 & 0 & 0 & 0 & 0 & 0 & 0 & 0.18045 & 0.191843 & 0.301659 & 0.008885 \\
\hline 62 & Phenol & 0.009874 & 0.004779 & 0.0131 & 0 & 0.002407 & 0 & 0 & 0 & 0 & 0 & 0 & 0 & 0 \\
\hline 63 & $\begin{array}{l}\text { 1H-pyrrole-2- } \\
\text { carboxaldehyde }\end{array}$ & 0.044414 & 0.031137 & 0.13181 & 0 & 0.004833 & 0.020459 & 0 & 0 & 0 & 0 & 0 & 0 & 0 \\
\hline 64 & Furaneol & 0.005049 & 0.00826 & 0 & 0 & 0 & 0 & 0 & 0 & 0 & 0 & 0 & 0 & 0 \\
\hline 65 & $\begin{array}{l}\text { 1,6-Octadiene-3, } \\
\text { 5-diol,3,7-dimethyl- }\end{array}$ & 0 & 0 & 0 & 0 & 0 & 0 & 0.122313 & 0.075155 & 0.029088 & 0.0449 & 0.037619 & 0.016984 & 0.018734 \\
\hline 66 & 4-Hexenoic acid & 0 & 0 & 0 & 0 & 0 & 0 & 0 & 0 & 0 & 0 & 0 & 0 & 0 \\
\hline 67 & Benzenemethamine & 0 & 0.005749 & 0 & 0.032418 & 0 & 0 & 0 & 0 & 0 & 0 & 0 & 0 & 0 \\
\hline 68 & $\begin{array}{l}\text { 2-Phenylethyl } \\
\text { benzoate }\end{array}$ & 0.002601 & 0.010949 & 0 & 0 & 0.013448 & 0 & 0.005596 & 0.001382 & 0.019374 & 0.04077 & 0 & 0 & 0 \\
\hline 69 & $\begin{array}{l}\text { 2H-Pyran-2-one, } \\
\text { tetrahydro-6-ethyl }\end{array}$ & 0 & 0 & 0 & 0 & 0.00249 & 0 & 0.006371 & 0.002313 & 0 & 0 & 0 & 0 & 0 \\
\hline 70 & $\begin{array}{l}\text { Undecanoid acid, } \\
\text { methyl ester }\end{array}$ & 0 & 0 & 0 & 0 & 0 & 0 & 0 & 0 & 0 & 0 & 0 & 0 & 0 \\
\hline 71 & Jasmin lactone & 0.087514 & 0.266736 & 0.45832 & 0.150783 & 0.106578 & 0.18588 & 0.309478 & 0.145746 & 0.081474 & 0.01239 & 0.093367 & 0.020351 & 0.031316 \\
\hline 72 & $\begin{array}{l}\text { 3-Hexen-1-ol, } \\
\text { formate,(Z)- }\end{array}$ & 0 & 0 & 0 & 0 & 0 & 0 & 0 & 0 & 0 & 0 & 0 & 0 & 0 \\
\hline 73 & Methylethylmaleimide & 0.012338 & 0.011889 & 0.00206 & 0 & 0 & 0 & 0.002747 & 0.001954 & 0 & 0.00748 & 0 & 0.001084 & 0 \\
\hline 74 & $\begin{array}{l}\text { 1H-Benzotriazole, } \\
\text { 1-ethenyl- }\end{array}$ & 0 & 0 & 0 & 0.491471 & 0 & 0 & 0 & 0 & 0.400174 & 0 & 0.01449 & 0 & 0 \\
\hline 75 & Dihydroactinidiolide & 0.006033 & 0.010195 & 0 & 0 & 0 & 0.036396 & 0 & 0 & 0 & 0 & 0 & 0 & 0 \\
\hline 76 & $\begin{array}{l}\text { 1,7-Octadien-3, } \\
\text { 6-diol,2,6-dimethyl- }\end{array}$ & 0 & 0 & 0 & 0 & 0 & 0 & 0.008524 & 0 & 0 & 0.05271 & 0 & 0 & 0 \\
\hline 77 & Methyl jasmonate & 0 & 0 & 0 & 0 & 0 & 0 & 0.00907 & 0 & 0 & 0.00168 & 0 & 0 & 0.001051 \\
\hline 78 & Coumaran & 0.023408 & 0 & 0.03534 & 0 & 0 & 0.015937 & 0 & 0 & 0 & 0 & 0 & 0 & 0 \\
\hline 79 & Coumarin & 0.002089 & 0 & 0 & 0 & 0 & 0.016262 & 0.013367 & 0.007711 & 0 & 0 & 0.002842 & 0.007176 & 0.001736 \\
\hline 80 & Indole & 0 & 0 & 0.09736 & 0.601278 & 0.433303 & 0.240362 & 1.119144 & 0.663919 & 1.057987 & 0.01336 & 0.48641 & 0.169741 & 0.453921 \\
\hline 81 & Phenol, 4-propyl- & 0 & 0 & 0 & 0 & 0 & 0 & 0 & 0 & 0 & 0 & 0 & 0 & 0 \\
\hline 82 & $\begin{array}{l}\text { Benzaldehyde, } \\
\text { 4-hydroxy- }\end{array}$ & 0 & 0 & 0 & 0 & 0 & 0 & 0 & 0 & 0 & 0 & 0 & 0 & 0 \\
\hline
\end{tabular}


Table S3. Volatiles determined in green tea.

\begin{tabular}{|c|c|c|c|c|c|c|c|c|}
\hline & & \multicolumn{7}{|c|}{ Green tea } \\
\hline & & TEA 14 & TEA 15 & TEA 16 & TEA 17 & TEA 18 & TEA 19 & TEA 20 \\
\hline 1 & Hexanal & 0 & 0 & 0 & 0 & 0 & 0 & 0 \\
\hline 2 & Undecan & 0 & 0 & 0 & 0.013286 & 0.011089 & 0.015404 & 0.01321 \\
\hline 3 & Methylpentenone derivative & 0 & 0 & 0 & 0 & 0 & 0 & 0 \\
\hline 4 & 1-Penten-3-ol & 0.017191 & 0.011164 & 0 & 0 & 0 & 0 & 0.005041 \\
\hline 5 & unknown 1 & 0 & 0 & 0 & 0 & 0 & 0 & 0 \\
\hline 6 & Methylbutanol derivative & 0.004415 & 0.00475 & 0 & 0.001923 & 0.004916 & 0.00629 & 0.004485 \\
\hline 7 & 2-Hexenal,(E)- & 0 & 0 & 0 & 0 & 0 & 0 & 0 \\
\hline 8 & 2,5-Dimethylpyrazine & 0 & 0 & 0 & 0 & 0 & 0 & 0 \\
\hline 9 & Diexthylbenzenederivative & 0.010145 & 0.013787 & 0 & 0.006512 & 0 & 0.005489 & 0.004517 \\
\hline 10 & 2,5-Dimethyl pyrazine & 0 & 0 & 0 & 0 & 0 & 0 & 0 \\
\hline 11 & 2-Penten-1-ol & 0.005605 & 0 & 0 & 0 & 0 & 0 & 0 \\
\hline 12 & Benzene,1,3-diethyl- & 0 & 0 & 0 & 0 & 0 & 0 & 0 \\
\hline 13 & 2-Ethyl pyrazine & 0 & 0 & 0 & 0 & 0 & 0 & 0 \\
\hline 14 & 1-Hexanol & 0 & 0 & 0.001165 & 0 & 0 & 0 & 0 \\
\hline 15 & 3-Hexen-1-ol & 0.006231 & 0.009791 & 0 & 0 & 0 & 0 & 0 \\
\hline 16 & 2-Hexen-1-ol, (E)- & 0 & 0 & 0 & 0 & 0 & 0 & 0 \\
\hline 17 & Pentylalcohol & 0.001363 & 0.002116 & 0.001638 & 0.000603 & 0.001012 & 0.00188 & 0 \\
\hline 18 & Linalool oxide I & 0.285221 & 0.356505 & 0.399367 & 0.361682 & 0.350891 & 0.363177 & 0.335858 \\
\hline 19 & 2-Furancarboxaldehyde & 0 & 0 & 0 & 0 & 0 & 0 & 0 \\
\hline 20 & 2,4-Heptadienal & 0 & 0 & 0 & 0 & 0 & 0 & 0 \\
\hline 21 & Furan,2,5-dimethyl & 0 & 0 & 0 & 0 & 0 & 0 & 0 \\
\hline 22 & Linalool oxide II & 0.001873 & 0.011113 & 0.003792 & 0 & 0 & 0 & 0 \\
\hline 23 & Furan-2-propyl & 0 & 0 & 0 & 0 & 0 & 0 & 0 \\
\hline 24 & Ethanone,1-(2-furanyl)- & 0 & 0 & 0 & 0 & 0 & 0 & 0 \\
\hline 25 & Benzenaldehyde & 0 & 0.001336 & 0.001879 & 0 & 0 & 0 & 0 \\
\hline 26 & Linalool & 0.003985 & 0.007335 & 0.003158 & 0 & 0 & 0 & 0.000263 \\
\hline 27 & 2-Furancarboxaldehyde,5-methyl- & 0 & 0 & 0 & 0 & 0 & 0 & 0 \\
\hline 28 & 2-Heptanone,5-methyl- & 0 & 0 & 0 & 0 & 0 & 0 & 0 \\
\hline 29 & 1H-pyrrole-2carboxaldehyde, & 0 & 0 & 0 & 0 & 0 & 0 & 0 \\
\hline 30 & 1,5,7-Octatrien-3-ol,3,7-dimethyl- & 0 & 0 & 0 & 0 & 0 & 0 & 0 \\
\hline 31 & Benzenamine,2-methoxy-5-methyl & 0 & 0 & 0 & 0 & 0 & 0 & 0 \\
\hline 32 & 2-Furanmethanol & 0 & 0 & 0 & 0 & 0 & 0 & 0 \\
\hline 33 & 2(3H)-furanone,5-ethyldihydro & 0 & 0 & 0 & 0 & 0 & 0 & 0 \\
\hline 34 & 2(3H)-furanone,5-ethyldihydro & 0 & 0 & 0 & 0 & 0 & 0 & 0 \\
\hline 35 & 4-Ethyl benzaldehyde & 0.015032 & 0.020284 & 0.02492 & 0.012545 & 0.00895 & 0.009528 & 0.0097 \\
\hline 37 & Butanoic acid, 2-methyl & 0 & 0 & 0 & 0 & 0 & 0 & 0 \\
\hline 38 & 1-Isopropyl-2-methoxy-4-methylbenzene & 0.054952 & 0.090987 & 0 & 0.035548 & 0.034376 & 0.023011 & 0.016856 \\
\hline 39 & Linalool oxide III & 0.01598 & 0.005968 & 0.004012 & 0.005581 & 0 & 0.002687 & 0.003841 \\
\hline
\end{tabular}




\section{Continued}

\begin{tabular}{|c|c|c|c|c|c|c|c|c|}
\hline 40 & Benzenamine,4-ethoxy- & 0 & 0 & 0 & 0 & 0 & 0 & 0 \\
\hline 41 & Linalool oxide IV & 0 & 0.037983 & 0.026404 & 0.055647 & 0.012717 & 0.016636 & 0.02001 \\
\hline 42 & Methyl salicylate & 0 & 0 & 0.001562 & 0 & 0 & 0 & 0 \\
\hline 43 & Nerol & 0 & 0 & 0 & 0 & 0 & 0 & 0 \\
\hline 44 & Benzoic acid,4-ethyl, methyl ester & 0.07397 & 0.118467 & 0.019743 & 0.053539 & 0.052089 & 0.032586 & 0.023506 \\
\hline 45 & 3,4-Dimethyl acetophenone & 0.034445 & 0.054957 & 0.050657 & 0.030412 & 0.023913 & 0.02371 & 0.020676 \\
\hline 46 & Benzoic acid,4-formyl-,methylester & 0.030777 & 0.049499 & 0.010073 & 0.025885 & 0.024246 & 0.015527 & 0.009943 \\
\hline 47 & O-Diacetylbenzene & 0.010382 & 0.018577 & 0 & 0.004793 & 0.004933 & 0 & 0 \\
\hline 48 & Geraniol & 0.05234 & 0.074953 & 0.004948 & 0 & 0 & 0 & 0 \\
\hline 49 & 4-Ethyl acetophenone & 0.041707 & 0.067988 & 0.046961 & 0.03902 & 0.019136 & 0.027503 & 0.022749 \\
\hline 50 & Benzyl alcohol & 0.038927 & 0.079246 & 0.021702 & 0.008251 & 0.003131 & 0.004859 & 0.004232 \\
\hline 51 & 2-Phenylethanol & 0.022699 & 0.045358 & 0.003618 & 0.001255 & 0.000744 & 0 & 0 \\
\hline 53 & Methyl cinamate & 0.007159 & 0.011558 & 0 & 0.004382 & 0.004184 & 0 & 0 \\
\hline 54 & Hexanoic acid & 0 & 0 & 0 & 0 & 0 & 0 & 0 \\
\hline 55 & Benzeneacetonitrile & 0 & 0 & 0 & 0 & 0 & 0 & 0 \\
\hline 56 & Jasmone & 0.005784 & 0.015073 & 0 & 0 & 0 & 0 & 0 \\
\hline 57 & Butanoic acid,3-hexenyl ester & 0 & 0 & 0.001572 & 0 & 0 & 0 & 0 \\
\hline 58 & 3,7-Octadien-2,6-diol,2,6-dimethyl- & 0 & 0 & 0 & 0 & 0 & 0 & 0 \\
\hline 59 & Ethanone,1-(1H-pyrrol-2-yl)- & 0.000823 & 0 & 0 & 0 & 0 & 0 & 0 \\
\hline 60 & 4-(1-hydroxyethyl)benzaldehyde & 0.013156 & 0.018623 & 0.027676 & 0.012013 & 0.009194 & 0.013278 & 0.00869 \\
\hline 61 & 4-Hydroxy-3-methylacetophenone & 0.01049 & 0.014641 & 0 & 0.008703 & 0.006552 & 0.009104 & 0.005837 \\
\hline 62 & Phenol & 0 & 0 & 0 & 0 & 0 & 0 & 0 \\
\hline 63 & 1H-pyrrole-2-carboxaldehyde & 0 & 0 & 0 & 0 & 0 & 0 & 0 \\
\hline 64 & Furaneol & 0 & 0 & 0 & 0 & 0 & 0 & 0 \\
\hline 65 & 1,6-Octadiene-3,5-diol,3,7-dimethyl- & 0 & 0 & 0 & 0 & 0 & 0 & 0 \\
\hline 66 & 4-Hexenoic acid & 0 & 0 & 0 & 0 & 0 & 0 & 0 \\
\hline 67 & Benzenemethamine & 0 & 0 & 0 & 0 & 0 & 0 & 0 \\
\hline 68 & 2-Phenylethyl benzoate & 0 & 0 & 0 & 0 & 0 & 0 & 0 \\
\hline 69 & 2H-Pyran-2-one,tetrahydro-6-ethyl & 0 & 0 & 0 & 0 & 0 & 0 & 0 \\
\hline 70 & Undecanoid acid, methyl ester & 0 & 0.005561 & 0 & 0.010156 & 0.013887 & 0.014884 & 0.008337 \\
\hline 71 & Jasmin lactone & 0.001613 & 0.002837 & 0.007933 & 0 & 0 & 0 & 0 \\
\hline 72 & 3-Hexen-1-ol,formate,(Z)- & 0 & 0 & 0.013315 & 0 & 0 & 0 & 0 \\
\hline 73 & Methylethylmaleimide & 0 & 0 & 0.00241 & 0 & 0 & 0 & 0 \\
\hline 74 & 1H-Benzotriazole,1-ethenyl- & 0 & 0 & 0 & 0 & 0 & 0 & 0 \\
\hline 75 & Dihydroactinidiolide & 0 & 0 & 0.003659 & 0 & 0 & 0 & 0 \\
\hline 76 & 1,7-Octadien-3,6-diol,2,6-dimethyl- & 0 & 0 & 0 & 0 & 0 & 0 & 0 \\
\hline 77 & Methyl jasmonate & 0 & 0 & 0 & 0 & 0 & 0 & 0 \\
\hline 78 & Coumaran & 0 & 0 & 0.001669 & 0 & 0 & 0 & 0 \\
\hline 79 & Coumarin & 0 & 0.000968 & 0.005591 & 0.018764 & 0.003012 & 0 & 0.003997 \\
\hline 80 & Indole & 0.020377 & 0.051619 & 0.049513 & 0.002644 & 0.004206 & 0.003602 & 0.044296 \\
\hline 81 & Phenol, 4-propyl- & 0 & 0 & 0 & 0 & 0 & 0 & 0 \\
\hline 82 & Benzaldehyde,4-hydroxy- & 0 & 0 & 0.022579 & 0 & 0 & 0 & 0 \\
\hline
\end{tabular}


Table S4. Volatiles determined in black tea.

\begin{tabular}{|c|c|c|c|c|c|c|c|c|c|c|c|c|c|c|c|c|c|c|}
\hline & \multicolumn{18}{|c|}{ Black tea } \\
\hline & TEA 21 & TEA 22 & TEA 23 & TEA 24 & 4 TEA 25 & TEA 26 & TEA 27 & TEA 28 & TEA 29 & TEA 30 & 0 TEA 31 & TEA 32 & TEA 33 & TEA 34 & TEA 35 & TEA 36 & TEA 37 & TEA 38 \\
\hline 1 Hexanal & 0.050283 & 30.093654 & 0.02275 & 0.019013 & 30.009955 & 50.013462 & 0 & 0.037265 & 0 & 0 & 0 & 0 & 0.018051 & 0 & 0 & 0 & 0 & 0 \\
\hline 2 Undecan & 0 & 0 & 0 & 0 & 0 & 0 & 0 & 0 & 0 & 0 & 0 & 0 & 0 & 0 & 0 & 0 & 0 & 0 \\
\hline $3 \begin{array}{l}\text { Methylpentenone } \\
\text { derivative }\end{array}$ & 0 & 0 & 0 & 0 & 0 & 0 & 0 & 0 & 0 & 0 & 0 & 0 & 0 & 0 & 0 & 0 & 0 & 0 \\
\hline 4 1-Penten-3-ol & 0.221826 & 0.1086970 & 0.054263 & 0 & 0.042266 & 0.03645 & 0.057938 & 0.117379 & 0.09006 & 0.072494 & 40.171505 & 50.076447 & 70.098218 & 0.021 & 0.02726 & 0.129086 & 0.084089 & 0.063793 \\
\hline 5 unknown 1 & 0 & 0 & 0 & 0 & 0 & 0 & 0 & 0 & 0 & 0 & 0 & 0 & 0 & 0 & 0 & 0 & 0 & 0 \\
\hline${ }_{6} \begin{array}{l}\text { Methylbutanol } \\
\text { derivative }\end{array}$ & 0.060309 & 0.012216 & 0 & 0 & 0.027676 & 0.021815 & 50.015059 & 0.058327 & 70.02234 & 0.018189 & 0 & 0 & 0 & 0.011 & 0.041633 & 30.030829 & 0.01785 & 0.010756 \\
\hline 7 2-Hexenal,(E)- & 0.304533 & 0.102849 & 0 & 0 & 0 & 0 & 0 & 0 & 0 & 0 & 0 & 0 & 0.103989 & 0 & 0 & 0 & 0 & 0 \\
\hline 8 2,5-Dimethylpyrazine & 0 & 0 & 0 & 0 & 0 & 0 & 0 & 0 & 0 & 0 & 0 & 0 & 0 & 0 & 0 & 0 & 0 & 0 \\
\hline $\begin{array}{l}9 \begin{array}{l}\text { Diexthylbenzene } \\
\text { derivative }\end{array} \\
\text { a }\end{array}$ & 0 & 0 & 0 & 0 & 0 & 0 & 0 & 0 & 0 & 0 & 0 & 0 & 0 & 0 & 0 & 0 & 0 & 0 \\
\hline $10 \begin{array}{l}2,5 \text {-Dimethyl } \\
\text { pyrazine }\end{array}$ & 0 & 0 & 0 & 0 & 0 & 0 & 0 & 0 & 0 & 0 & 0 & 0 & 0 & 0 & 0 & 0 & 0 & 0 \\
\hline 11 2-Penten-1-ol & 0.349069 & 0.0676580 & 0.024233 & 30.016819 & 90.03806 & 0.022797 & 70.067319 & 0.095468 & 30.068516 & 0.071106 & 60.106472 & 20.034418 & 80.049442 & 0.0155 & 0.018473 & 30.094426 & 0.0578 & 0.03536 \\
\hline 12 Benzene,1, 3-diethyl- & 0 & 0 & 0 & 0 & 0 & 0 & 0 & 0 & 0 & 0 & 0 & 0 & 0 & 0 & 0 & 0 & 0 & 0 \\
\hline 13 2-Ethyl pyrazine & 0 & 0 & 0 & 0 & 0 & 0 & 0 & 0 & 0 & 0 & 0 & 0 & 0 & 0 & 0 & 0 & 0 & 0 \\
\hline 14 1-Hexanol & 0.056901 & 10.036568 & 0 & 0.011389 & 90.027039 & 0.014769 & 90.01231 & 0.038591 & 10.055546 & 0.05197 & 70.052052 & 20.017145 & 50.022088 & 0.0219 & 0.038706 & $60.106503 c$ & 0.051764 & 40.036871 \\
\hline 153-Hexen-1-ol & 0.68145 & 0.1708990 & 0.019385 & 50.053407 & 70.144201 & 10.063563 & 30.085587 & 0.281916 & 50.140618 & 0.125208 & 80.243671 & 0.108932 & 20.120272 & 0.1868 & 0.173864 & 40.5455730 & 0.191814 & 40.135573 \\
\hline 16 2-Hexen-1-ol, (E)- & 0.086734 & 40.050134 & 0 & 0 & 0.026137 & 70.012984 & 40.017542 & 0.102118 & 30.125691 & 0.088498 & 80.136779 & 0.027993 & 30.039083 & 0.0322 & 0.012939 & 90.2273290 & 0.067095 & 50.099996 \\
\hline 17 Pentylalcohol & 0 & 0 & 0 & 0 & 0 & 0.003327 & 70.00361 & 0.003237 & 70.001981 & 0 & 0.008257 & 70.004993 & 30.004286 & 0.0057 & 0.004501 & 10.0049070 & 0.005546 & 0.006962 \\
\hline 18 Linalool oxide I & 1.421303 & 30.908019 & 0.52101 & 0.441741 & 10.542082 & 20.508451 & 10.735071 & 1.233934 & 40.359785 & 0.38176 & $6 \quad 0.91989$ & 0.48981 & 0.526897 & 0.5464 & 0.897974 & 41.88714 & 0.783807 & 0.729617 \\
\hline 19 2-Furancarboxaldehyde & 0 & 0 & 0 & 0 & 0 & 0 & 0 & 0 & 0 & 0 & 0 & 0 & 0 & 0.0167 & 0 & 0 & 0 & 0 \\
\hline 20 2,4-Heptadienal & 0 & 0.0394950 & 0.015084 & 0 & 0 & 0 & 0 & 0.00757 & 0 & 0 & 0 & 0.005969 & 90.025793 & 0 & 0 & 0 & 0 & 0 \\
\hline 21 Furan,2,5-dimethyl & 0 & 0 & 0 & 0.024266 & 0 & 0 & 0 & 0 & 0 & 0 & 0 & 0 & 0 & 0 & 0 & 0.029264 & 0 & 0.011259 \\
\hline 22 Linalool oxide II & 1.013935 & 0.3360410 & 0.080694 & 40.191938 & 80.460204 & 40.373073 & 30.39009 & 0.954359 & 0.056171 & 0.043468 & 80.255203 & 0.162615 & 50.159972 & 0.341 & 0.903061 & 12.002691 & 0.84779 & 0.460012 \\
\hline 23 Furan-2-propyl & 0 & 0.0206680 & 0.005399 & 90.00417 & 0 & 0 & 0 & 0.006767 & 0 & 0 & 0 & 0.00592 & 0.014718 & 0 & 0.002775 & $50.010959 c$ & 0.004107 & 70.006941 \\
\hline $24 \begin{array}{l}\text { Ethanone, } \\
1 \text {-(2-furanyl)- }\end{array}$ & 0 & 0 & 0 & 0 & 0 & 0 & 0 & 0 & 0 & 0 & 0 & 0 & 0 & 0 & 0 & 0 & 0 & 0 \\
\hline 25 Benzenaldehyde & 0.052889 & 0.1766590 & 0.011528 & 80.033005 & 50.018196 & 0.01962 & 0.045454 & 0.074875 & 50.005205 & 0.004967 & 70.03405 & 0.012672 & 20.020547 & 0.0115 & 0.052299 & 90.0484530 & 0.026628 & 30.020711 \\
\hline 26 Linalool & 0.183418 & 30.0616730 & 0.013959 & 90.012666 & 60.395096 & 0.213822 & 20.440133 & 0.0957 & 0.149427 & 0.106838 & 80.261172 & 20.135233 & 30.069957 & 0.158 & 0.21579 & 0.6257650 & 0.279275 & 50.076368 \\
\hline $\begin{array}{l}27 \text {-Furancarboxaldehyde, } \\
\text { 5-methyl- }\end{array}$ & 0 & 0 & 0 & 0.009288 & 0 & 0 & 0 & 0 & 0 & 0 & 0 & 0 & 0 & 0 & 0 & 0.009857 & 0 & 0.00267 \\
\hline 28 2-Heptanone,5-methyl- & 0 & 0 & 0 & 0 & 0 & 0 & 0 & 0 & 0 & 0 & 0 & 0 & 0 & 0 & 0 & 0 & 0 & 0 \\
\hline $29 \begin{array}{l}\text { 1H-pyrrole- } \\
\text { 2carboxaldehyde, }\end{array}$ & 0 & 0 & 0 & 0 & 0.01197 & 0.020797 & 0 & 0 & 0 & 0 & 0 & 0 & 0 & 0 & 0 & 0.018912 & 0 & 0 \\
\hline $\begin{array}{l}30 \text { 1,5,7-Octatrien-3-ol,3, } \\
\text { 7-dimethyl- }\end{array}$ & 0.25257 & 0.015052 & 0.02273 & 0.017818 & 80.023619 & 0.043578 & 80.083637 & 0.262481 & 0 & 0 & 0.001884 & 0 & 0 & 0.0136 & 0.005206 & 60.1925910 & 0.043988 & 0.018029 \\
\hline $\begin{array}{l}\text { Benzenamine, } \\
\text { 2-methoxy-5-methyl }\end{array}$ & 0 & 0 & 0 & 0 & 0 & 0 & 0 & 0 & 0 & 0 & 0 & 0 & 0 & 0 & 0 & 0 & 0 & 0 \\
\hline 32 2-Furanmethanol & 0.005845 & 0 & 0 & 0.024004 & 0 & 0.013261 & 10.007284 & 0 & 0 & 0 & 0.008218 & 0 & 0 & 0.0077 & 0 & 0.032194 & 0 & 0.01831 \\
\hline $\begin{array}{l}\text { 2(3H)-furanone, } \\
\text { 5-ethyldihydro }\end{array}$ & 0 & 0 & 0 & 0 & 0 & 0 & 0 & 0 & 0 & 0 & 0 & 0 & 0 & 0 & 0.024685 & 50.027189 & 0 & 0 \\
\hline 34 2(3H)-furanone, & 0.221295 & 0.0175530 & 0.014349 & 90.009645 & 0 & 0 & 0 & 0 & 0 & 0 & 0 & 0 & 0.008058 & 0 & 0 & 0 & 0 & 0 \\
\hline 35 4-Ethyl benzaldehyde & 0.003557 & 0 & 0 & 0 & 0 & 0 & 0 & 0 & 0 & 0 & 0 & 0 & 0.006924 & 0 & 0.015621 & $10.009966 \mathrm{C}$ & 0.017499 & 0.024888 \\
\hline 37 Butanoic acid, 2-methyl & 0 & 0 & 0 & 0 & 0.015269 & 0.010993 & 0 & 0 & 0 & 0 & 0 & 0 & 0 & 0 & 0 & 0 & 0 & 0 \\
\hline $\begin{array}{l}38 \text { 1-Isopropyl-2-methoxy } \\
\text {-4-methylbenzene }\end{array}$ & 0 & 0.010727 & 0 & 0.024325 & 0 & 0 & 0 & 0 & 0 & 0.003718 & 0 & 0 & 0.013112 & 0.0133 & 0.034859 & 90.030887 & 0 & 0 \\
\hline 39 Linalool oxide III & 0.27328 & 0.2278250 & 0.064895 & 50.007336 & 60.133178 & 30.120907 & 70.203789 & 0.45679 & 0.003523 & 0.00301 & 10.020806 & 0.00877 & 0.015719 & 0.0282 & 0.107531 & 10.1519160 & 0.112499 & 0.055263 \\
\hline $\begin{array}{l}\text { 40 Benzenamine, } \\
\text { 4-ethoxy- }\end{array}$ & 0 & 0 & 0 & 0 & 0 & 0 & 0 & 0 & 0 & 0 & 0 & 0 & 0 & 0 & 0 & 0 & 0 & 0 \\
\hline
\end{tabular}




\section{Continued}

\begin{tabular}{|c|c|c|c|c|c|c|c|c|c|c|c|c|c|c|c|c|c|c|}
\hline 41 Linalool oxide IV & 0.400065 & 0.314051 & 510.09242 & 0.2340960 & 0.377233 & 0.371979 & 90.338915 & 0.389507 & 0.0134190 & 0.012608 & 0.09257 & 0.052481 & 10.065295 & 50.1057 & 70.361725 & 50.417372 & 20.274188 & 80.14112 \\
\hline 42 Methyl salicylate & 0.341064 & 0.008798 & 980.005093 & 30.0063170 & 0.035978 & 0.035617 & 70.344129 & 0.038024 & 0.0339460 & 0.022326 & 0.085551 & 10.213584 & 40.070652 & 20.0452 & 20.074226 & 60.280135 & 50.078688 & 380.070898 \\
\hline 43 Nerol & 0.007634 & 0 & 0 & 0 & 0 & 0 & 0 & 0 & 0 & 0 & 0 & 0 & 0 & 0 & 0 & 0 & 0 & 0 \\
\hline $\begin{array}{l}\text { 44 Benzoic acid,4-ethyl, } \\
\text { methyl ester }\end{array}$ & 0.034652 & 0.018349 & 190.010655 & 50.0360140 & 0.016726 & 0.018068 & 80.01528 & 0.021509 & 0.002780 & 0.005191 & 10.020954 & 40.009456 & 60.022513 & 30.0127 & 70.02655 & 50.039468 & 80.008323 & 30.013059 \\
\hline $\begin{array}{l}45^{\text {3,4-Dimethyl }} \text { acetophenone }\end{array}$ & 0.034671 & 0.014419 & 190.010484 & 40.0190220 & 0.018986 & 0.013704 & 40.013407 & 0.015588 & 0.0092410 & 0.012663 & 30.024304 & 40.011816 & 60.027815 & 50.0138 & 80.042523 & 30.044568 & 80.036923 & 230.056455 \\
\hline $\begin{array}{l}46 \\
\text { Benzoic acid,4-formyl-, } \\
\text { methylester }\end{array}$ & 0.01268 & 0.006884 & 0 & 0.0239850 & 0.006635 & 0.007701 & 10.006375 & 0.010466 & 0 & 0 & 0.009514 & 40.004802 & 20.009317 & 70.4446 & 60.010626 & 60.016358 & 0 & 0.006815 \\
\hline 47 O-Diacetylbenzene & 0 & 0 & 0 & 0 & 0 & 0 & 0 & 0 & 0 & 0 & 0 & 0 & 0 & 0 & 0 & 0 & 0 & 0 \\
\hline 48 Geraniol & 1.260518 & 0.043723 & 230.035917 & 70.1007540 & 0.756134 & 40.846676 & 60.048052 & 0.392736 & 0 & 0 & 0.030293 & 30.014303 & 30.008994 & 0 & 0.476999 & 90.661557 & 70.204581 & 310.245601 \\
\hline 49 4-Ethyl acetophenone & 0.030387 & 0.016643 & 430.010363 & 0 & 0.020332 & 0.012543 & 30.012889 & 0.017052 & 0.0071920 & 0.011467 & 70.023453 & 30.01187 & 70.024516 & 60.0141 & 10.034743 & 30.042477 & 70.033361 & 10.048638 \\
\hline 50 Benzyl alcohol & 1.210593 & 1.47216 & $\begin{array}{ll}6 & 0.233963\end{array}$ & 0.734092 & 0.47722 & 0.56876 & 0.576994 & 1.173924 & 0.1013890 & 0.080222 & 20.15104 & 0.050872 & 20.126068 & 80.1284 & 40.344552 & 20.502736 & 60.259551 & 10.392265 \\
\hline 51 2-Phenylethanol & 1.997018 & 1.096002 & 20.327 & 0.6105521 & 1.678485 & 1.256742 & 20.276295 & 0.936773 & 0.1771870 & 0.143328 & 30.202683 & 30.038435 & 50.055666 & 60.1268 & 80.414552 & 20.609475 & 50.281816 & 60.193228 \\
\hline 53 Methyl cinamate & 0 & 0 & 0 & 0 & 0 & 0 & 0 & 0 & 0 & 0 & 0 & 0 & 0 & 0 & 0 & 0 & 0 & 0 \\
\hline 54 Hexanoic acid & 0.255744 & 1.481868 & 0 & 0 & 0.145494 & 0.120495 & 50.036914 & 0.085249 & 0 & 0 & 0.258387 & 70.187449 & 90.086971 & 0 & 0.157828 & 80.208848 & 80.123217 & 0 \\
\hline 55 Benzeneacetonitrile & 0 & 0.051786 & 0 & 0 & 0 & 0 & 0 & 0 & 0 & 0 & 0 & 0 & 0 & 0 & 0 & 0 & 0 & 0 \\
\hline 56 Jasmone & 0 & 0 & 0 & 0 & 0 & 0 & 0 & 0 & 0 & 0 & 0 & 0 & 0 & 0 & 0 & 0 & 0 & 0 \\
\hline $\begin{array}{l}57 \begin{array}{l}\text { Butanoic acid, } \\
\text { 3-hexenyl ester }\end{array}\end{array}$ & 0.047871 & 0.028818 & 180.01865 & 0 & 0 & 0 & 0.01376 & 0.03272 & 0 & 0 & 0 & 0 & 0 & 0 & 0 & 0 & 0 & 0 \\
\hline $\begin{array}{l}58 \text { 3,7-Octadien-2,6-diol, } \\
\text { 2,6-dimethyl- }\end{array}$ & 0 & 0 & 0 & 0 & 0 & 0 & 0 & 0 & 0 & 0 & 0 & 0 & 0 & 0 & 0 & 0 & 0 & 0 \\
\hline $\begin{array}{l}59 \text { Ethanone, } \\
\text { 1-(1H-pyrrol-2-yl)- }\end{array}$ & 0.035699 & 0 & 0.00802 & 0.055288 & 0.02063 & 0.037997 & 70.012331 & 0.009871 & 0 & 0 & 0.035819 & 0 & 0 & 0.0318 & 80.024081 & 10.084593 & 30.016088 & 880.034741 \\
\hline $\begin{array}{l}60 \text { 4-(1-hydroxyethyl) } \\
\text { benzaldehyde }\end{array}$ & 0 & 0 & 0 & 0 & 0 & 0 & 0 & 0 & 0 & 0 & 0 & 0 & 0 & 0 & 0 & 0 & 0 & 0 \\
\hline $\begin{array}{l}61 \text { 4-Hydroxy-3- } \\
\text { methylacetophenone }\end{array}$ & 0 & 0 & 0 & 0 & 0 & 0 & 0 & 0 & 0 & 0 & 0 & 0 & 0 & 0 & 0 & 0 & 0 & 0 \\
\hline 62 Phenol & 0 & 0 & 0 & 0 & 0 & 0.00397 & 0.006189 & 0 & 0 & 0 & 0 & 0 & 0 & 0 & 0 & 0 & 0 & 0 \\
\hline $63 \begin{array}{l}1 \mathrm{H} \text {-pyrrole-2 } \\
\text {-carboxaldehyde }\end{array}$ & 0.008994 & 0 & 0 & 0.0842890 & 0.013804 & 0.036317 & 70.012636 & 0.008509 & 0 & 0 & 0.003622 & 0 & 0 & 0.0138 & 80.027124 & 40.02584 & 0.003969 & 90.011776 \\
\hline 64 Furaneol & 0 & 0 & 0 & 0 & 0 & 0 & 0 & 0 & 0 & 0 & 0 & 0 & 0 & 0 & 0 & 0.017884 & 0 & 0 \\
\hline 65 1,6-Octadiene-3, & 0 & 0 & 0 & 0 & 0 & 0 & 0 & 0 & 0 & 0 & 0 & 0 & 0 & 0 & 0 & 0 & 0 & 0 \\
\hline 66 4-Hexenoic acid & 2.204504 & 0.223613 & 30.394017 & 0 & 0.566074 & 0 & 0.182465 & 0 & 0 & 0 & 0 & 0 & 0 & 1.1489 & 91.450741 & 11.308687 & 0 & 0.579922 \\
\hline 67 Benzenemethamine & 0 & 0 & 0 & 0 & 0 & 0 & 0 & 0 & 0 & 0 & 0 & 0 & 0 & 0 & 0 & 0 & 0 & 0 \\
\hline 68 2-Phenylethyl benzoate & 0 & 0 & 0 & 0 & 0 & 0 & 0 & 0 & 0 & 0 & 0 & 0 & 0 & 0 & 0 & 0 & 0 & 0 \\
\hline $\begin{array}{l}69 \\
\text { tetrahydro-6-ethyl }\end{array}$ & 0 & 0 & 0 & 0 & 0 & 0 & 0 & 0 & 0 & 0 & 0 & 0 & 0 & 0 & 0 & 0 & 0 & 0 \\
\hline $\begin{array}{l}70 \text { Undecanoid acid, } \\
\text { methyl ester }\end{array}$ & 0.043795 & 0.029795 & 50.016643 & 0 & 0 & 0 & 0 & 0 & 0 & 0 & 0 & 0 & 0 & 0 & 0 & 0 & 0 & 0 \\
\hline 71 Jasmin lactone & 0 & 0.009082 & 320.003701 & 10.005857 & 0 & 0 & 0 & 0 & 0 & 0 & 0 & 0 & 0 & 0 & 0 & 0.006491 & 0 & 0 \\
\hline $72 \begin{array}{l}\text { 3-Hexen-1-ol, } \\
\text { formate,(Z)- }\end{array}$ & 0 & 0 & 0 & 0 & 0 & 0 & 0 & 0 & 0 & 0 & 0 & 0 & 0 & 0 & 0 & 0 & 0 & 0 \\
\hline 73 Methylethylmaleimide & 0 & 0 & 0 & 0 & 0 & 0 & 0 & 0 & 0 & 0 & 0 & 0 & 0.005608 & 0 & 0 & 0 & 0 & 0 \\
\hline $\begin{array}{l}74 \text { 1-ethenyl- } \\
\text { 1H-Benzotriazole, }\end{array}$ & 0 & 0 & 0 & 0 & 0 & 0 & 0 & 0 & 0 & 0 & 0 & 0 & 0 & 0 & 0 & 0 & 0 & 0 \\
\hline 75 Dihydroactinidiolide & 0 & 0 & 0.008384 & 0 & 0 & 0 & 0 & 0 & 0 & 0 & 0 & 0.008396 & 60.015198 & 0 & 0.015543 & 0 & 0 & 0 \\
\hline 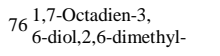 & 0 & 0.01333 & 0 & 0 & 0 & 0 & 0.000585 & 0 & 0 & 0 & 0 & 0 & 0 & 0 & 0 & 0 & 0 & 0 \\
\hline 77 Methyl jasmonate & 0 & 0 & 0 & 0 & 0 & 0 & 0 & 0 & 0 & 0 & 0 & 0 & 0 & 0 & 0 & 0 & 0 & 0 \\
\hline 78 Coumaran & 0 & 0 & 0.002577 & 70.0040310 & 0.002005 & 0.004051 & 10.004994 & 0.00267 & 0 & 0 & 0 & 0 & 0 & 0.0091 & 10.004341 & 10.018345 & 0 & 0.006257 \\
\hline 79 Coumarin & 0 & 0 & 0 & 0 & 0 & 0 & 0 & 0 & 0 & 0 & 0 & 0 & 0 & 0 & 0 & 0.040482 & 0 & 0 \\
\hline 80 Indole & 0 & 0.012561 & 10.001285 & 0 & 0 & 0 & 0 & 0 & 0 & 0 & 0 & 0 & 0.001363 & 0 & 0 & 0 & 0 & 0 \\
\hline 81 Phenol, 4-propyl- & 0 & 0 & 0 & 0 & 0 & 0 & 0 & 0 & 0 & 0 & 0 & 0 & 0 & 0.0136 & 60.01508 & 0 & 0 & 0 \\
\hline $\begin{array}{l}\text { Benzaldehyde, } \\
\text { 4-hydroxy- }\end{array}$ & 0 & 0 & 0.019254 & 40.021435 & 0 & 0 & 0 & 0 & 0 & 0 & 0 & 0 & 0 & 0 & 0.060349 & 0 & 0 & 0 \\
\hline
\end{tabular}


Scientific Research Publishing (SCIRP) is one of the largest Open Access journal publishers. It is currently publishing more than 200 open access, online, peer-reviewed journals covering a wide range of academic disciplines. SCIRP serves the worldwide academic communities and contributes to the progress and application of science with its publication.

Other selected journals from SCIRP are listed as below. Submit your manuscript to us via either submit@scirp.org or Online Submission Portal.
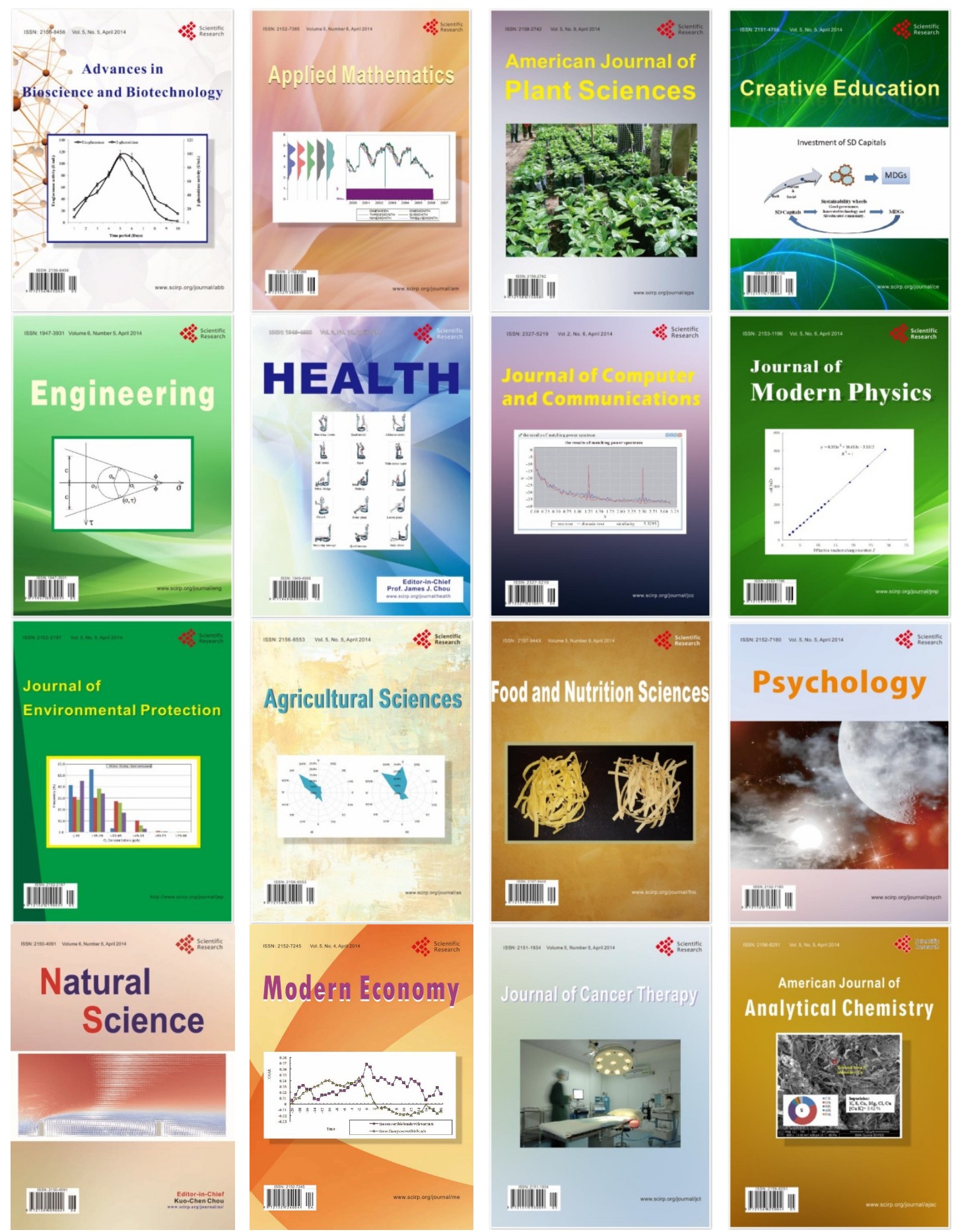